\title{
The Potential Functions of the Methyl Halides
}

\author{
Z. I. Slawsky and David M. Dennison \\ University of Michigan, Ann Arbor, Michigan
}

(Received May 1, 1939)

\begin{abstract}
The problem of the potential functions of the methyl halides is re-examined in an attempt to find a function which is both adequate and simple. A valence form of potential was tried which contained four constants: $k_{1}$, the $\mathrm{C}-\mathrm{H}$ elongation; $c$, the $\mathrm{C}-\mathrm{X}$ elongation; $k_{2}$, the deformation of the $\mathrm{H}-\mathrm{C}-\mathrm{H}$ angle; and $k_{3}$, the deformation of the $\mathrm{X}-\mathrm{C}-\mathrm{H}$ angle. It was found that this simple valence potential must be modified by the inclusion of a cross product term between the $\mathrm{X}-\mathrm{C}$ distance and the
\end{abstract}

$\mathrm{X}-\mathrm{C}-\mathrm{H}$ angle, thus introducing a fifth constant, $k_{\text {. }}$. The constants $k_{1}$ and $k_{2}$ were determined from the methane frequencies $\left(k_{1}=4.88 \times 10^{5}\right.$ and $\left.k_{2}=0.443 \times 10^{5}\right)$ and were taken to be the same for all the methyl halides. By adjusting the three remaining constants it was possible to predict eight quantities, the six fundamental frequencies and two of the fine structure spacings. The agreement with the observed values was satisfactory, the average deviation being less than 1 percent.
$\mathrm{T}$

HE infra-red spectra of the methyl halides were investigated by Bennett and Meyer $^{1}$ in 1928 and since then a number of attempts have been made to obtain a potential energy function which will adequately describe these molecules. Sutherland and Dennison ${ }^{2}$ have treated the parallel motions of $\mathrm{FCH}_{3}, \mathrm{ClCH}_{3}, \mathrm{BrCH}_{3}$ and $\mathrm{ICH}_{3}$ while Voge and Rosenthal ${ }^{3}$ have considered the vibrations of the $\mathrm{ClCH}_{3}$ molecule. In both cases the authors have chosen potential functions with as many or nearly as many arbitrary constants as could be determined by means of the available experimental data. In this paper the normal vibrations of these molecules are reexamined in an attempt to find a potential function which is physically plausible, simple, and yet adequate for the explanation of the observed bands and their fine structure spacings.

The geometrical configuration of these molecules has already been described in the preceding paper. In assuming a potential function we were guided by the following considerations: It is well known from the work of Rosenthal on methane, ${ }^{4}$ Howard on ammonia, ${ }^{5}$ and Cross and Van Vleck on water $^{6}$ that these molecules, possessing a heavy atom together with several hydrogen atoms, have potential fields closely approxi-

${ }^{1}$ W. H. Bennett and C. F. Meyer, Phys, Rev, 32, 888 (1928)

${ }_{2}$ G. B. B. M. Sutherland and D. M. Dennison, Proc. Roy. Soc. 148, 250 (1935).

${ }^{3}$ H. H. Voge and J. E. Rosenthal, J. Chem. Phys. 4, 137 (1936).

${ }^{4}$ J. E. Rosenthal, Phys. Rev. 45, 538 (1934).

5 J. B. Howard, J. Chem. Phys. 3, 207 (1935).

6 J. H. Van Vleck and P. C. Cross, J. Chem. Phys, 1, 357 (1933). mating the so-called valence type. That is, the potential energy depends only on the distance of each hydrogen atom from the heavy atom and upon the angles between the bond directions. Thus in the case of methane the potential energy may be given by two constants, $k_{1}$, which determines the resistance to the stretching of the carbon-hydrogen bond and $k_{2}$, which gives the resistance to the deformation of the $\mathrm{H}-\mathrm{C}-\mathrm{H}$ angle. It is also well known that molecules containing two or more heavy atoms, as for example $\mathrm{CCl}_{4}$, cannot be treated by means of a valence type potential. We have therefore made two assumptions about the force field of the methyl halides. First, that the methyl group is bound by the valence type forces, the same for all the members of the series, and in fact, identical with those occurring in methane. Secondly, that the forces which bind the halogen atom to the methyl group are of a more general type.

In accordance with the above assumptions each methyl halide molecule requires five force constants for its potential energy function. Two of these, $k_{1}$ and $k_{2}$, have already been mentioned. The third constant describes the change in the potential energy when the halogen-carbon bond is stretched, while the fourth constant measures the energy change for a deformation of the $\mathrm{X}-\mathrm{C}-\mathrm{H}$ angles. The fifth gives the interaction term between the last two, that is, it indicates the change in the resistance to the deformation of the $\mathrm{X}-\mathrm{C}-\mathrm{H}$ angle when the $\mathrm{X}-\mathrm{C}$ bond is stretched, and vice versa. The last three constants will be designated by $c, k_{3}$ and $k_{4}$, respectively. Since $k_{1}$ 
and $k_{2}$ are adjusted to satisfy the methane frequencies, only the last three constants have to be evaluated from the normal frequencies of the methyl halides.

Each methyl halide has six fundamental bands. Three of these suffice for the adjustment of the three remaining constants and hence there are three more to act as checks on our assumptions. Aside from this, when the potential energy constants are known, one may calculate the fine structure of the perpendicular bands. This fine structure spacing is very sensitive to these constants and therefore gives us another good test for the assumed potential functions.

The assumed potential energy is of the form:

$$
\begin{aligned}
2 V= & k_{1} \sum_{i}\left(\delta r_{i}\right)^{2}+k_{2} \sum_{i}\left(\delta \alpha_{i}\right)^{2} \\
& +k_{3} r_{0}{ }^{2} \sum_{i}\left(\delta \beta_{i}\right)^{2}+c\left(\delta x_{0}\right)^{2}+2 k_{4} r_{0}\left(\delta x_{0}\right) \sum_{i}\left(\delta \beta_{i}\right),
\end{aligned}
$$

where $i$ takes on the values $1,2,3$, corresponding to the three hydrogen atoms in the methyl group. The coordinates are illustrated in Fig. $1 . x_{0}$ is the $\mathrm{X}-\mathrm{C}$ distance at equilibrium, $r_{0}$ the $\mathrm{H}-\mathrm{C}$ distance, $\beta_{i}$ the $\mathrm{X}-\mathrm{C}-\mathrm{H}$ angle and $\alpha_{i}$ the $\mathrm{H}-\mathrm{C}-\mathrm{H}$ angle. $\delta r_{i}$ corresponds then to the stretching of the $\mathrm{C}-\mathrm{H}$ bond, $\delta \alpha_{i}$ the deformation in the $\mathrm{H}-\mathrm{C}-\mathrm{H}$ angle, $\delta x_{0}$ the change in the distance from carbon to the halogen atom and $\delta \beta_{i}$ the change in the $\mathrm{X}-\mathrm{C}-\mathrm{H}$ angle.

In a paper by Rosenthal and Voge ${ }^{7}$ the most general potential functions for the parallel and perpendicular frequencies of the $\mathrm{ZYX}_{3}$ molecule, the kinetic energy and the resulting cubic equations, which determine the frequencies, are explicitly written out. We shall therefore obtain the relationship between their general force constants and ours and thus arrive at the cubic equations in terms of our potential constants.

The cubic equation for the parallel frequencies as given in their paper is

$$
\lambda_{11}{ }^{3}+R_{1} \lambda_{11}{ }^{2}+R_{2} \lambda_{11}+R_{3}=0,
$$

where the $R$ 's are functions of the force constants $G_{i j}$ of the potential energy function

$$
\begin{aligned}
2 V_{\mathrm{II}}=G_{11} s_{1}{ }^{2}+G_{22} s_{2}{ }^{2} & +G_{33} s_{3}{ }^{2} \\
& +2 G_{12} s_{1} s_{2}+2 G_{13} s_{1} s_{3}+2 G_{23} s_{2} s_{3} .
\end{aligned}
$$

${ }_{7}^{7}$ J. E. Rosenthal and H. H. Voge, J. Chem. Phys, 4, 134 $(1936)$.

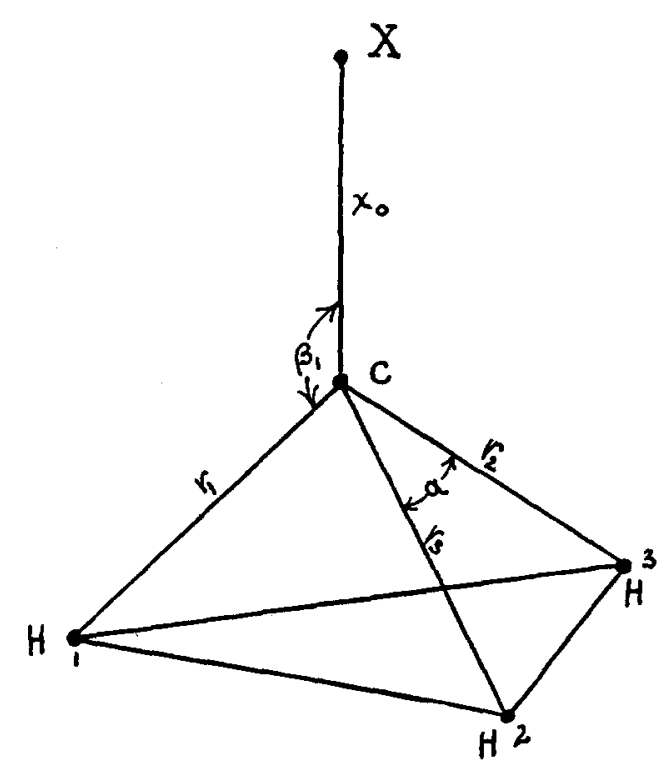

FIG. 1.

It is convenient to give the $\lambda$ 's in such units that $\lambda_{i}=4 \pi^{2} \nu_{i}{ }^{2} m c_{L}{ }^{2}$ where $m$ is the mass of an hydrogen atom. The cubic for the perpendicular frequencies is :

$$
\lambda_{\perp}{ }^{3}+P_{1} \lambda_{\perp}{ }^{2}+P_{2} \lambda_{\perp}+P_{3}=0
$$

and again the $P$ 's are functions of the $G_{k l}$ 's of the potential energy expression

$$
\begin{aligned}
2 V_{\perp}=G_{44} s_{4}{ }^{2}+G_{55} s_{5}{ }^{2} & +G_{66} s_{6}{ }^{2} \\
& +2 G_{45} s_{4} s_{5}+2 G_{56} s_{5} s_{6}+2 G_{46} s_{4} s_{6} .
\end{aligned}
$$

These functions are given explicitly in the papers mentioned.

The coordinate system that is used by Rosenthal and Voge is illustrated in Fig. 2. $r_{i}$ is the distance of any hydrogen atom from the carbon atom, $r_{4}$ is the $\mathrm{X}-\mathrm{C}$ distance, $q_{i j}$ the $\mathrm{H}-\mathrm{H}$ distance, $q_{i 4}$ the $\mathrm{X}-\mathrm{H}$ distance, $\theta$ the $\mathrm{H}-\mathrm{C}$ to $\mathrm{X}-\mathrm{C}$ angle and $\psi$ the angle made by the $\mathrm{X}-\mathrm{H}$ line and the $\mathrm{X}-\mathrm{C}$ line. The $s_{j}$ 's which appear in the potential functions are then defined in terms of the above-described coordinates by the following relations:

$$
\begin{array}{ll}
s_{1}=\delta r_{1}+\delta r_{2}+\delta r_{3}, & s_{4}=\delta r_{2}-\delta r_{3}, \\
s_{2}=\delta r_{4}, & s_{5}=\delta q_{24}-\delta q_{34}, \\
s_{3}=\delta q_{12}+\delta q_{13}+\delta q_{23}, & s_{6}=\delta q_{12}-\delta q_{13} .
\end{array}
$$

The evaluation of the $G$ 's in terms of the con- 
stants $k_{1}, k_{2}, k_{3}, c$ and $k_{4}$ is straight forward and yields the following relations:

$$
\begin{aligned}
& G_{11}=\frac{1}{3}\left(k_{1}+8 k_{2}+8 k_{3}\right) ; \quad G_{12}=2 \sqrt{2} k_{4} ; \\
& G_{22}=c ; \\
& G_{23}=-\sqrt{ } 3 k_{4} ; \\
& G_{33}=k_{2}+k_{3} ; \quad G_{31}=-\frac{2 \sqrt{ } 6}{3}\left(k_{2}+k_{3}\right) ; \\
& G_{44}=\frac{k_{1}}{2}+k_{2}+\frac{(2 \sqrt{2}+\cot \psi)^{2}}{2(2 \sqrt{2} \cot \psi-1)^{2}} k_{3} ; \\
& G_{45}=-\frac{3}{2} \frac{(2 \sqrt{2}+\cot \psi)}{\sin ^{2} \psi(2 \sqrt{2} \cot \psi-1)^{2}} k_{3} ; \\
& G_{55}=\frac{9}{2} \frac{k_{3}}{\sin \psi(2 \sqrt{2} \cot \psi-1)^{2}} ; \quad G_{46}=-\left(\frac{3}{2}\right)^{\frac{1}{2}} k_{2} ; \\
& G_{66}=\frac{3}{2} k_{2} ; \quad G_{56}=0 .
\end{aligned}
$$

We may substitute these into the expressions for the $P$ 's and $R$ 's as given by Rosenthal and Voge ${ }^{8}$ and thus obtain the cubic equations for the parallel and perpendicular frequencies in terms of our potential force constants. The coefficients are :

$$
R_{1}=\frac{37}{36} k_{1}+\frac{11}{9}\left(k_{2}+k_{3}\right)+\frac{12+M_{2}}{12 M_{2}} c-\frac{\sqrt{2}}{3} k_{4},
$$

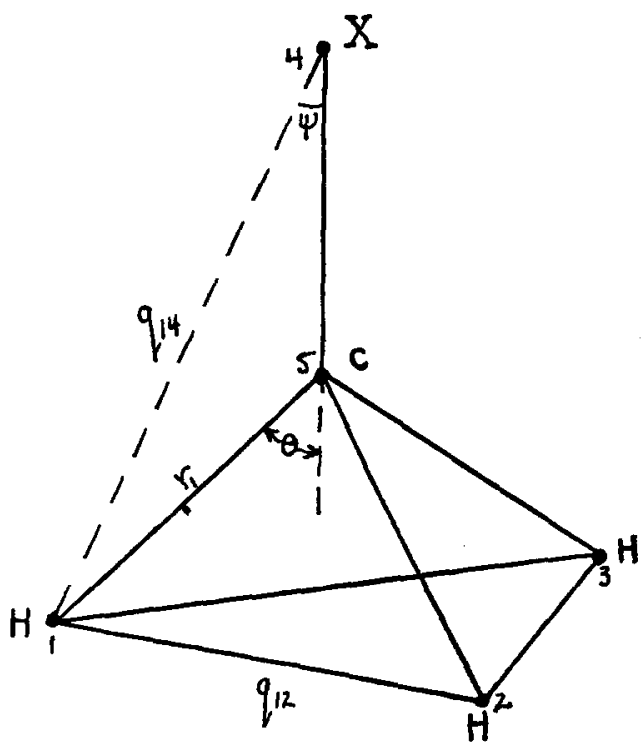

FIG. 2.

${ }^{8}$ J. E. Rosenthal and H. H. Voge, J. Chem. Phys. 4, 134 (1936).

$$
\begin{gathered}
R_{2}=-\frac{5}{4} k_{1}\left(k_{2}+k_{3}\right)+\frac{c}{12 M_{2}} \\
\quad \times\left\{\left(\frac{37}{3}+M_{2}\right) k_{1}+\left(\frac{44}{3}+M_{2}\right)\left(k_{2}+k_{3}\right)\right\} \\
\quad-\left(\frac{11}{3 M_{2}} k_{4}{ }^{2}+\frac{1}{4} k_{4}{ }^{2}+\frac{\sqrt{2}}{3} k_{1} k_{4}\right), \\
R_{3}=\frac{c}{12 M_{2}}\left(15+M_{2}\right) k_{1}\left(k_{2}+k_{3}\right)-\frac{15+M_{2}}{4 M_{2}} k_{1} k_{4}{ }^{2}, \\
P_{1}=\frac{1}{9}\left(10 k_{1}+\frac{49}{2} k_{2}\right) \\
\quad+\frac{8}{9}(2 \sqrt{2} \sin \psi+\cos \psi)^{2}+\frac{27}{2 M_{2}} \sin ^{2} \psi \\
(2 \sqrt{2} \cos \psi-\sin \psi)^{2}
\end{gathered}
$$

$P_{2}=\frac{1}{2}\left\{k_{1} k_{2}+\frac{2 k_{3}\left(k_{1}+2 k_{2}\right)}{(2 \sqrt{2} \cos \psi-\sin \psi)^{2}}\right.$

$$
\left.+\cos \psi \frac{(2 \sqrt{2} \sin \psi+\cos \psi)}{(2 \sqrt{2} \cos \psi-\sin \psi)^{2}} k_{2} k_{3}\right\}
$$$$
+\frac{3}{2 M_{2}} \frac{\sin ^{2} \psi\left(k_{1}+2 k_{2}\right) k_{3}}{(2 \sqrt{2} \cos \psi-\sin \psi)^{2}}
$$

$$
\begin{aligned}
& +\frac{27}{2 M_{2}} \frac{\sin ^{2} \psi\left(k_{1}+\frac{5}{2} k_{2}\right) k_{3}}{(2 \sqrt{2} \cos \psi-\sin \psi)^{2}}+k_{1} k_{3}+\frac{5}{2} k_{1} k_{2} \\
& -\frac{9(2 \sqrt{2} \sin \psi+\cos \psi)^{2}}{4} k_{2} k_{3}
\end{aligned}
$$

$$
+\frac{81}{4} \frac{k_{2} k_{3}}{(2 \sqrt{2} \cos \psi-\sin \psi)^{2}},
$$

$$
\begin{aligned}
P_{3}=\frac{27}{8} & \left\{\frac{2}{3}(2 \sqrt{2} \cos \psi-\sin \psi)^{2}\right. \\
& \left.+\sin ^{2} \psi\left(\frac{12}{M_{2}}-\frac{2}{3}\right)+\frac{4}{3}\right\} \frac{k_{1} k_{2} k_{3}}{(2 \sqrt{2} \cos \psi-\sin \psi)^{2}} .
\end{aligned}
$$

By assigning observed values to the $\lambda$ 's appearing in these equations we may evaluate the constants.

The values of $k_{1}$ and $k_{2}$, which are to be chosen so as to best fit the methane frequencies, are evaluated as follows: In the above expressions we 
set $M_{2}=1, \psi=\theta / 2, k_{3}=k_{2}, c=k_{1}$ and $k_{4}=0$, and identify those frequencies which correspond to the parallel frequencies of the $Z_{Y X}$ molecule and those that correspond to the perpendicular. The $R$ 's and the $P$ 's then become:

$$
\begin{array}{ll}
R_{1}=\frac{1}{9}\left(19 k_{1}+22 k_{2}\right), & P_{1}=\frac{1}{9}\left(10 k_{1}+49 k_{2}\right), \\
R_{2}=\frac{1}{9}\left(10 k_{1}{ }^{2}+46 k_{1} k_{2}\right), & P_{2}=6 k_{1} k_{2}+(22 / 3) k_{2}{ }^{2}, \\
R_{3}=(8 / 3) k_{1}{ }^{2} k_{2}, & P_{3}=8 k_{1} k_{2}{ }^{2} .
\end{array}
$$

Dennison $^{9}$ has shown that the methane frequencies $\nu_{1}, \nu_{3}, \nu_{4}$ correspond to the parallel frequencies of the $Z_{Y X}$ molecule and hence should satisfy Eq. (1), when the proper values of $k_{1}$ and $k_{2}$ are taken. $\nu_{2}, \nu_{3}, \nu_{4}$ should come out of Eq. (2), since they correspond to the perpendicular frequencies of the $Z_{Y} \mathrm{YX}_{\mathbf{3}}$ molecule.

For $k_{1}=4.878 \times 10^{5}$ dynes/centimeter, and $k_{2}=0.443 \times 10^{5}$ dynes/centimeter, we obtain the values given in the Table $I$. We have also given the experimentally observed values to show what order of approximation is involved when the methane molecule is treated by means of a valence type potential function.

The discrepancy between the observed and calculated normal frequencies of methane may be attributed to two factors. In the first place, the calculated frequencies were obtained by means of a very simple type of potential function, involving only two arbitrary constants, $k_{1}$ and $k_{2}$. It is very probable that such a picture does not give the exact nature of the binding forces. In the second place, one must consider the anharmonicity corrections. Any calculation of normal frequencies assumes that the atoms of the molecule may be considered as simple harmonic oscillators. For small amplitudes of vibration of these atoms about their positions of equilibrium, this approximation is good, but will become increasingly bad with greater amplitudes. Thus even the most general potential function for methane, involving five arbitrary constants, may not give the normal frequencies of heavy methane within a percent of the observed values.

In evaluating the remaining constants of the methyl halides, that is, $k_{3}, c$ and $k_{4}$, one may notice that only $k_{1}, k_{2}$ and $k_{3}$ appear in the expressions for the perpendicular frequencies of these molecules. One chooses $k_{3}$ so that equation

${ }^{9}$ D. M. Dennison, Rev. Mod. Phys. 3, 208 (1931).
TABLE I.

\begin{tabular}{ccc}
\hline Band & Calculated & OBSERVED \\
\hline \multicolumn{3}{c}{ Parallel Frequencies } \\
$\nu_{1}$ & 2876 & 2915 \\
$\nu_{3}$ & 3050 & 3014 \\
$\nu_{5}$ & 1324 & 1304 \\
& Perpendicular Frequencies \\
$\nu_{2}$ & 3050 & 3014 \\
$\nu_{4}$ & 1495 & 1520 \\
$\nu_{6}$ & 1324 & 1304 \\
\hline
\end{tabular}

(2) gives the correct value for $\nu_{6}$, rather than the other perpendicular frequencies, because it is the most sensitive to the value of $k_{3}$. The other two roots of this equation may now be used to check against the other two observed perpendicular frequencies.

Once $k_{3}$ is determined, $c$ and $k_{4}$ are adjusted to fit $\nu_{5}$ and $\nu_{3}$ in Eq. (1). The remaining root is then checked against the remaining observed parallel frequency. In the numerical work we set $m=1$, $M_{1}=12$ and $M_{2}$ equal to the atomic weight of iodine, bromine, chlorine or fluorine as the case may be. For the carbon-halogen distance, $x_{0}$, a mean value as given by infra-red and electron diffraction data ${ }^{10}$ was used. This distance of course determines the angle $\psi$, since the dimensions of the methyl group are assumed to be the same as those of methane. It was found that the values of the force constants were not very sensitive to this angle.

\section{$\mathrm{ICH}_{3}$}

Consider the methyl-iodide molecule. Since the binding forces of the methyl group are taken to be those of methane, the dimensions of this group are also taken from methane as given by Johnston and Dennison. ${ }^{11}$ Thus $r_{0}$, the $\mathrm{H}-\mathrm{C}$ distance, is $1.11 \times 10^{-8} \mathrm{~cm}$. The total height of this molecule, from the iodine atom to the plane of the three hydrogen atoms, is about $1.75 \times 10^{-8}$ $\mathrm{cm}$. This value is not very accurate but the force constants are quite insensitive to this dimension.

When these constants are used in Eq. (2) it becomes :

$$
\begin{aligned}
\lambda_{\perp}{ }^{3}-\left(6.625+1.096 k_{3}\right) \lambda_{\perp}{ }^{2} & \\
& +\left(6.481+7.156 k_{3}\right) \lambda_{\perp}-6.714 k_{3}=0,
\end{aligned}
$$

${ }^{10}$ L. O. Brockway, Rev. Mod. Phys. 8, 231 (1936).

11 M. Johnston and D. M. Dennison, Phys. Rev. 48, 868 (1935). 
TABle II.

\begin{tabular}{c|c|c|c}
\hline BAND & Calculated & Observed & \% Deviation \\
\hline$\nu_{2}$ & 3034 & 3074 & 1.3 \\
$\nu_{4}$ & 1445 & 1446 & 0.1 \\
$\nu_{6}$ & $886^{*}$ & 886 & \\
$\nu_{1}$ & 2916 & 2916 & 0.0 \\
$\nu_{3}$ & $1252^{*}$ & 1252 & \\
$\nu_{5}$ & $532^{*}$ & 532 & \\
\hline
\end{tabular}

a cubic in $\lambda$. The constant $k_{3}$ may be considered as an adjustable parameter, to be so determined as to give one of the observed frequencies. We do this as follows: The $\nu_{6}$ fundamental frequency of $\mathrm{ICH}_{3}$ is 886 waves $\mathrm{cm}^{-1}$. Since we define $\lambda$ to be $4 \pi^{2} m c_{L}^{2} v^{2}$ where $c_{L}$ is the velocity of light, $\nu$ the frequency in waves $\mathrm{cm}^{-1}$ and $m$ the mass of the hydrogen atom, its value is $0.4641 \times 10^{5}$ dynes $/ \mathrm{cm}$. This is inserted into the above cubic equation and the resulting equation is then solved for $k_{3}$. The value thus obtained is $0.463 \times 10^{5}$ dynes $/ \mathrm{cm}$. If our assumed potential energy function is a correct one, once this constant is determined, the roots of Eq. (2) must then correspond to the three normal perpendicular frequencies of this molecule. In Table II we have tabulated the observed and calculated frequencies. The starred frequencies are those used for the adjustment of our constants, so that they do not serve as checks.

The constants $c$ and $k_{4}$, are obtained from Eq. (1). To do this we must use two observed frequencies. $\nu_{5}$ and $\nu_{3}$ are observed to be 532 and 1252 waves $/ \mathrm{cm}$., respectively. The corresponding $\lambda$ 's are then 0.1671 and 0.9257 . When these values are put into Eq. (1) there result two equations in $c$ and $k_{4}$, which may be treated simultaneously. It should be noticed that these equations are quadratic in $k_{4}$. Upon solving them, one obtains the two values: $k_{4}=0.786 \pm 0.134$. The occurrence of two roots is of course due to the mathematical method employed since physically only one such value can be correct. To determine which of these is the appropriate one, one must turn to entirely different considerations. The two values of $k_{4}$ will give two different values for $c$. Since $c$ measures the strength of the bond between the methyl group and the halogen atom, we may get an approximate value for it by assuming that one of the normal frequencies corresponds to the motion of the methyl group against the halogen atom. Sutherland and Dennison $^{2}$ have calculated the constant $c$ on such an assumption. Comparison with their results shows that the $c$ corresponding to $k_{4}$ equal to $0.652 \times 10^{5} \mathrm{dynes} / \mathrm{cm}$ is the correct one. The other value comes out to be entirely too high. The value we adopt therefore is $3.292 \times 10^{5}$. With these constants determined one may solve Eq. (1) and obtain the three $\nu$ 's which correspond to the three parallel normal frequencies. These are tabulated in Table II together with the experimentally observed values.

The agreement between the calculated and observed frequencies is on the whole very satisfactory. One must of course remember that the values which act as checks are not the ones most sensitive to the force constants. Fortunately there is another independent check on the force constants which determine the perpendicular motions. When such constants are known one may calculate the fine structure spacing of these bands and compare them with the experimental results of Bennett and Meyer. ${ }^{1}$ This calculation is presented later in this paper.

\section{$\mathrm{BrCH}_{3}$}

Next let us consider the methyl bromide molecule. The distance from the bromine atom to the plane of the hydrogen atoms is $2.28 \times 10^{-8}$ $\mathrm{cm}$. The atomic weight of the bromine atom is taken as 79.92. The cubic equation for the perpendicular frequencies then becomes:

$$
\begin{aligned}
\lambda_{\perp}{ }^{3}-\left(6.625+1.111 k_{3}\right) \lambda_{\perp}{ }^{2} & \\
& +\left(6.481+7.250 k_{3}\right) \lambda_{\perp}-6.816 k_{3}=0 .
\end{aligned}
$$

The observed $\nu_{6}$ for this molecule is $957 \mathrm{~cm}^{-1}$ so that $\lambda_{6}=0.5407 \times 10^{5}$. This value of $\lambda$ gives $k_{3}=0.536 \times 10^{5}$. Putting this into the expression for the parallel bands one obtains:

$$
\begin{aligned}
& \lambda_{l 1}{ }^{3}-\left(6.209+0.096 c-0.471 k_{4}\right) \lambda_{l 1}{ }^{2} \\
& +\left(5.966+0.566 c-2.299 k_{4}-0.296 k_{4}{ }^{2}\right) \lambda_{11} \\
& -0.472 c+1.448 k_{4}{ }^{2}=0
\end{aligned}
$$

The $\lambda$ 's corresponding to the observed $\nu_{5}$ and $\nu_{3}$ are 0.2197 and 1.0072 , respectively. The solution of the two simultaneous equations arising from these, again gives two roots for $k_{4} ;$ viz. $k_{4}=0.798$ \pm 0.023 . For reasons already discussed under $\mathrm{ICH}_{3}$ we chose the smaller value and the corre- 
sponding value of $c$ is 4.193 . The calculated frequencies as well as the observed ones are listed in Table III.

\section{$\mathrm{ClCH}_{3}$}

The fact that the two values of $k_{4}$ for $\mathrm{BrCH}_{3}$ are more nearly alike than the two values for $\mathrm{ICH}_{3}$ does not appear to be purely accidental, as will become apparent when we consider methyl chloride and methyl fluoride.

The chlorine atom is $2.07 \times 10^{-8} \mathrm{~cm}$ from the plane of the hydrogen atoms. The atomic weight of chlorine is taken to be 35.4 so that the expression for the perpendicular bands becomes:

$\lambda_{\perp}{ }^{3}-\left(6.625+1.140 k_{3}\right) \lambda_{\perp}{ }^{2}$

$$
+\left(6.481+7.435 k_{3}\right) \lambda_{1}-7.010 k_{3}=0 \text {. }
$$

From the above equation $k_{3}=0.5964$ when $\lambda$ is set equal to 0.6143 . With this value for $k_{3}$ the expression for the parallel bands becomes:

$$
\begin{aligned}
& \lambda_{11}{ }^{3}-\left(6.283+0.112 c-0.471 k_{4}\right) \lambda_{\mathrm{t}}{ }^{2} \\
& +\left(6.337+0.671 c-2.299 k_{4}-0.354 k_{4}{ }^{2}\right) \lambda \\
& -0.601 c+1.736 k_{4}{ }^{2}=0 .
\end{aligned}
$$

The observed $\lambda$ 's are $\lambda_{3}=0.3164$ and $\lambda_{5}=1.0842$. It turns out that only complex values of $k_{4}$ will satisfy the two equations resulting from the two $\lambda$ 's, simultaneously. This of course indicates a flaw in our assumed form of the potential function. It can, however, be readily shown that this flaw is not a serious one. For successive members of this methyl halide series the parabolae which represent the possible values of $k_{4}$ lie increasingly higher with respect to the zero axis. Thus in the case of $\mathrm{ICH}_{3}$ the two roots are further apart than in the case of $\mathrm{BrCH}_{3}$, while for $\mathrm{ClCH}_{3}$ this parabola does not cross the axis at all. We may take the point of nearest approach of the parabola to the axis as the root and see how well such a value of $k_{4}$ gives the frequencies. The $c$ corresponding to the real part of $k_{4}=0.725$

TABLE III.

\begin{tabular}{c|c|c|c}
\hline \hline BAND & Calculated & ObSERVEd & \% Deviation \\
\cline { 2 - 3 }$\nu_{2}$ & 3034 & 3062 & 0.9 \\
$\nu_{4}$ & 1450 & 1450 & 0.0 \\
$\nu_{6}$ & $957^{*}$ & 957 & \\
$\nu_{1}$ & 2915 & 2917 & 0.0 \\
$\nu_{3}$ & $1306^{*}$ & 1306 & \\
$\nu_{5}$ & $610^{*}$ & 610 & \\
\hline
\end{tabular}

TABLE IV.

\begin{tabular}{c|c|c|c}
\hline \hline Band & Calculated & ObSERved & \% Deviation \\
\cline { 2 - 3 }$\nu_{2}$ & 3035 & 3047 & 0.4 \\
$\nu_{4}$ & 1456 & 1460 & 0.3 \\
$\nu_{6}$ & $1020^{*}$ & 1020 & \\
$\nu_{1}$ & 2916 & 2923 & 0.3 \\
$\nu_{3}$ & 1363 & 1355 & 0.7 \\
$\nu_{5}$ & $732^{*}$ & 732 & \\
\hline
\end{tabular}

$\pm 0.173 i$ is 4.417 . The calculated $\nu^{\prime}$ s are given in Table IV. We take $k_{4}$ to be $0.725 \times 10^{5}$.

It is apparent from the above table that even though we get complex roots for $k_{4}$, our assumptions cannot be far from the truth. The behavior of the constant $k_{4}$ may be ascribed to the two factors already mentioned in the discussion of the methane molecule; namely, that the corrections for anharmonicity are neglected and the fact that the assumed form of the potential energy function is not entirely correct.

\section{$\mathrm{FCH}_{3}$}

As would be expected from the study of $\mathrm{ICH}_{3}$, $\mathrm{BrCH}_{3}$ and $\mathrm{ClCH}_{3}$, the parabola for the possible values of $k_{4}$ of $\mathrm{FCH}_{3}$ will approach the zero axis less closely than in the case of $\mathrm{ClCH}_{3}$. This turns out to be the case. To fix the constant $k_{3}$ we have taken $1.75 \times 10^{-8} \mathrm{~cm}^{-}$to be distance of the fluorine atom from the plane of the hydrogen atoms. The atomic weight used was 19.00 . With $\lambda_{6}=0.8504$ corresponding to the observed $\nu_{6}$ of $1200 \mathrm{~cm}^{-1}$ the expression :

$$
\begin{aligned}
\lambda_{\perp}{ }^{3}-\left(6.625+1.213 k_{3}\right) \lambda_{\perp}{ }^{2} \\
+\left(6.481+7.905 k_{3}\right) \lambda_{\perp}-7.495 k_{3}=0
\end{aligned}
$$

gives $k_{3}=0.8097 \times 10^{5}$.

The expression for the parallel frequencies:

$$
\begin{aligned}
& \lambda_{\mathrm{I1}}{ }^{3}-\left(6.544+0.136 c-0.471 k_{4}\right) \lambda_{11}{ }^{2} \\
& \quad+\left(7.637+0.855 c-2.299 k_{4}-0.443 k_{4}{ }^{2}\right) \lambda_{11} \\
& -0.911 c+2.182 k_{4}{ }^{2}=0
\end{aligned}
$$

will give two equations in $k_{4}$ and $c$ when $\lambda$ is set successively equal to 0.6495 and 1.2865 corresponding to the observed $\nu_{5}$ and $\nu_{3}$, respectively. Solving these simultaneously for $k_{4}$, one obtains the complex values $0.713 \pm 0.344 i$. Assigning to $k_{4}$ its real part, 0.713, $c$ comes out to be 6.091. In Table $\mathrm{V}$ appear the frequencies calculated with these constants. 
TABLE V.

\begin{tabular}{c|c|c|c}
\hline Band & Calculated & Observed & \% Deviation \\
\hline$\nu_{2}$ & 3037 & 2987 & 1.7 \\
$\nu_{4}$ & 1489 & 1476 & 1.0 \\
$\nu_{6}$ & $1200^{*}$ & 1200 & \\
$\nu_{1}$ & 2917 & 2914 & 0.1 \\
$\nu_{3}$ & 1517 & 1476 & 2.8 \\
$\nu_{5}$ & $1049^{*}$ & 1049 & \\
\hline
\end{tabular}

\section{Fine Structure of the Perpendicular} BANDS*

The perpendicular bands of the methyl halides show the usual anomalous fine structure that is found in the axially symmetrical molecules such as $\mathrm{NH}_{3}$ and spherically symmetrical molecules such as methane. This anomaly arises from the interaction of the degenerate vibratory motions and the angular momenta of the molecule.

Johnston and Dennison ${ }^{11}$ have shown how the vibration-rotation interaction may be calculated when the dimensions and the force constants of the molecule are known. Following their method we shall calculate the fine structure spacing of the perpendicular bands of $\mathrm{ICH}_{3}, \mathrm{BrCH}_{3}, \mathrm{ClCH}_{3}$, and $\mathrm{FCH}_{3}$, using the force constants obtained above.

That the perpendicular frequencies of axially symmetrical molecules are doubly degenerate is evident from the fact that the molecular forces are isotropic in a plane perpendicular to the axis of symmetry.

A proper choice of phases and amplitudes of two such harmonic vibrations at right angles to each other will give a circular motion. The angular momentum vector of this rotation is clearly in the direction of the symmetry axis. Each atom contributes to this internal angular momentum as it vibrates about its position of equilibrium. The total contribution may therefore be written as

$$
\begin{aligned}
& M_{2}\left(x_{5} \dot{y}_{5}-\dot{x}_{5} y_{5}\right)+M_{1}\left(x_{4} \dot{y}_{4}-\dot{x}_{4} y_{4}\right) \\
& \quad+3 m\left(x_{123} \dot{y}_{123}-\dot{x}_{123} y_{123}\right)-3 m\left(\xi \dot{\eta}-\dot{\xi}_{\eta}\right) .
\end{aligned}
$$

The first three terms are the respective angular

* A calculation of the $\zeta$ 's for the methyl halides was made somewhat earlier by Professors W. F. Colby and David M. Dennison using slightly different coordinates. Their formulae, which were not published, are in agreement with ours and we wish to make acknowledgment of this earlier calculation. momenta of the halogen atom, the carbon atom and the center of gravity of the hydrogen atoms about the axis of symmetry. The last term represents the sum of the angular momenta of each hydrogen atom about an axis through its equilibrium position and parallel to the symmetry axis. The negative sign that precedes this term is due to the fact that the angular momenta of the hydrogen atoms is opposite in direction to that of $Z, Y$ and $G$. (See Johnston and Dennison. ${ }^{11}$ )

The eigenvalues for the above expression are readily obtained when it is expressed in terms of normal coordinates, since then the wave functions are the usual Hermitian polynomials.

The relation between the coordinates used in the expression (3) and the normal coordinates $Q_{2}, Q_{4}, \cdots$ etc. are

$$
\begin{aligned}
x_{5}=\left(\rho_{51} A_{2}+\rho_{52} D_{2}\right) Q_{2} & \\
& +\left(\rho_{51} A_{4}+\rho_{52} D_{4}\right) Q_{4}+\left(\rho_{51} A_{6}+\rho_{52} D_{6}\right) Q_{6}, \\
x_{4}=\left(\rho_{41} A_{2}+\rho_{42} D_{2}\right) Q_{2} & \\
& +\left(\rho_{41} A_{4}+\rho_{42} D_{4}\right) Q_{4}+\left(\rho_{41} A_{6}+\rho_{42} D_{6}\right) Q_{6} \\
x_{123}=\left(\rho_{31} A_{2}\right. & \left.+\rho_{32} D_{2}\right) Q_{2} \\
& +\left(\rho_{31} A_{4}+\rho_{32} D_{4}\right) Q_{4}+\left(\rho_{31} A_{6}+\rho_{32} D_{6}\right) Q_{6},
\end{aligned}
$$

with identical expressions for $y_{5}, y_{4}$, and $y_{123}$, except that $Q_{2}, Q_{4}$ and $Q_{6}$ are replaced by $R_{2}, R_{4}$ and $R_{6}$, normal coordinates perpendicular to $Q_{2}$, $Q_{4}$ and $Q_{6}$, respectively. $A_{2}, A_{4}, A_{6} \cdots D_{6}$ have already been given explicitly in the preceding paper. The $\rho$ 's are defined as follows:

$$
\begin{aligned}
\rho_{51}= & \frac{\left[\left(\left(3 m+M_{1}\right) / 2\right) C_{0}+3 m M_{1} b_{0}^{2}\right]}{A_{0}\left(3 m+M_{1}+M_{2}\right)}, \\
\rho_{52}= & \frac{\left[\left(\left(3 m+M_{1}\right) / 2\right) a_{0} C_{0} / b_{0}-M_{2} C_{0} / 2\right]}{A_{0}\left(3 m+M_{1}+M_{2}\right)}, \\
\rho_{41}= & \frac{M_{2}\left(3 a_{0} b_{0} m+C_{0} / 2\right)}{A_{0}\left(3 m+M_{1}+M_{2}\right)}, \\
\rho_{42}= & \frac{\left(a_{0} M_{2} / b_{0}-M_{2}-3 m\right) C_{0} / 2}{A_{0}\left(3 m+M_{1}+M_{2}\right)}, \\
\rho_{31}= & \frac{M_{2}\left(a_{0} b_{0} M_{1}-b_{0} M_{1}-C_{0} / 2\right)}{A_{0}\left(3 m+M_{1}+M_{2}\right)} \\
\rho_{32}= & \frac{\left(M_{1}+a_{0} M_{2} / b_{0}\right) C_{0} / 2}{A_{0}\left(3 m+M_{1}+M_{2}\right)} .
\end{aligned}
$$


TABLE VI.

\begin{tabular}{c|c|c|c|c}
\hline \hline$\alpha$ & $\mathrm{FCH}_{3}$ & $\mathrm{ClCH}_{3}$ & $\mathrm{BrCH}_{3}$ & $\mathrm{ICH}_{3}$ \\
\hline$\alpha$ & 0.79 & 0.38 & 0.27 & 0.19 \\
$\beta$ & 16.32 & 14.05 & 13.40 & 12.62 \\
$\gamma$ & 2.83 & 2.79 & 2.81 & 2.81 \\
$\delta$ & 2.60 & 1.56 & 1.24 & 0.98 \\
$\epsilon$ & -0.188 & -0.112 & -0.090 & -0.071 \\
$\varphi$ & 2.28 & 2.44 & 2.49 & 2.55 \\
\hline
\end{tabular}

In terms of the normal coordinates, expression (3) becomes

$$
\begin{aligned}
\sum_{j}\left(Q_{j} \dot{R}_{j}-\dot{Q}_{j} R_{j}\right)\left\{A_{j}{ }^{2}\left(M_{2} \rho_{51}{ }^{2}+M_{1} \rho_{41}{ }^{2}+3 m \rho_{31}{ }^{2}\right)\right. \\
+2 A_{i} D_{j}\left(M_{2} \rho_{51} \rho_{52}+M_{1} \rho_{41} \rho_{42}+3 m \rho_{31} \rho_{32}\right) \\
\left.+D_{j}^{2}\left(M_{2} \rho_{52}{ }^{2}+M_{1} \rho_{42}{ }^{2}+3 m \rho_{32}{ }^{2}\right)-3 m B_{j}^{2}\right\}
\end{aligned}
$$

Since the wave functions are the well-known Hermitian orthogonal functions, the eigenvalues $\hbar \zeta$ for the first excited states of the bands $\nu_{i}$ are immediately given by

$$
\begin{aligned}
& A_{i}{ }^{2}\left(M_{2} \rho_{51}{ }^{2}+M_{1} \rho_{41}{ }^{2}+3 m \rho_{31}{ }^{2}\right) \\
& +2 A_{i} D_{i}\left(M_{2} \rho_{51} \rho_{52}+M_{1} \rho_{41} \rho_{42}+3 m \rho_{31} \rho_{32}\right) \\
& \quad+D_{i}{ }^{2}\left(M_{2} \rho_{52}{ }^{2}+M_{1} \rho_{42}{ }^{2}+3 m \rho_{32}{ }^{2}\right)-3 m B_{i}{ }^{2} .
\end{aligned}
$$

Johnston and Dennison have derived the expression for the fine structure spacing $\Delta \nu_{i}$ in terms of the $\zeta$ 's. They give it as (in waves per $\mathrm{cm})$ :

$$
\Delta \nu_{i}=\frac{\hbar}{c_{L}}\left(\frac{1-\zeta_{i}}{C_{0}}-\frac{1}{A_{0}}\right) .
$$

To obtain numerical values for the above, one must have the general force constants $\alpha, \beta, \gamma, \delta, \epsilon$, and $\varphi$, which may be readily obtained from the valence type force constants $k_{1}, k_{2}$ and $k_{3}$ already discussed in the earlier part of this paper. The
TABle VII.

\begin{tabular}{l|c|c|c|c|c|c}
\hline & \multicolumn{2}{|c|}{$\Delta \nu_{2}$} & \multicolumn{2}{c|}{$\Delta p_{4}$} & \multicolumn{2}{c}{$\Delta \nu_{6}$} \\
\hline & CALC. & OBS. & CALC. & OBS. & CALC. & OBS. \\
\hline $\mathrm{FCH}_{3}$ & 6.9 & 7.5 & 12.0 & 11.5 & 5.5 & 5.6 \\
$\mathrm{ClCH}_{3}$ & 7.7 & 8.2 & 12.9 & 12.0 & 6.4 & 7.0 \\
$\mathrm{BrCH}_{3}$ & 8.0 & 9.0 & 13.2 & 11.9 & 6.9 & 7.4 \\
$\mathrm{ICH}_{3}$ & 8.1 & 9.0 & 13.2 & 11.8 & 7.2 & 7.7 \\
\hline
\end{tabular}

transformation equations are:

$$
\begin{aligned}
\alpha & =\frac{3 r_{0}^{2} k_{3}}{2\left(a_{0}-b_{0}\right)^{2}}, & \delta & =\frac{4 r_{0} k_{3}}{a_{0}-b_{0}}, \\
\beta & =\frac{4}{3}\left(k_{1}+2 k_{2}+8 k_{3}\right), & \epsilon & =-\frac{1}{2 \sqrt{ } 3} \frac{r_{0} k_{3}}{a_{0}-b_{0}},
\end{aligned}
$$

$\gamma=\frac{4}{9}\left(k_{1}+\frac{25}{2} k_{2}+\frac{1}{2} k_{3}\right), \quad \varphi=\frac{4}{3 \sqrt{ } 3}\left(k_{1}-\frac{5}{2} k_{2}-k_{3}\right)$,

and from these one obtains for the general force constants the values given in Table VI.

In comparing the observed and calculated $\Delta \nu^{\prime}$ s (Table VII), actual differences rather than percentages should be considered because the spacings are small quantities both numerically and experimentally. The experimental values are taken from the work of Bennett and Meyer and are the mean values of about fifteen to twenty lines. The individual spacings vary considerably so that these values are to be considered good only within a few tenths of a wave per $\mathrm{cm}$. Though there seems to be a systematic deviation of the calculated values from the observed, we feel that they are small enough to indicate that the force constants are very nearly correct. Of course, the fine structure spacing of these perpendicular bands depends only on the force constants which enter into the perpendicular frequencies so that they act as a check on $k_{1}, k_{2}$, and $k_{3}$ only. 